\title{
Accidentes y lesiones por consumo de alcohol y drogas en pacientes atendidos en una sala de urgencia
}

\author{
Nora Nelly Oliva Rodríguez ${ }^{1}$ \\ Maria Célia Barcellos Dalrí2 \\ María Magdalena Alonso Castillo ${ }^{3}$ \\ Karla Selene López García ${ }^{4}$
}

Los propósitos del estudio fueron describir la diferencia del consumo de alcohol por género, escolaridad y ocupación y conocer el efecto de las características sociodemográficas sobre el consumo de alcohol, en 120 pacientes. Los resultados mostraron diferencias significativas por género $(t=-3,570, p<0,01)$ y escolaridad $(t=2,636, p<0,01)$ en el consumo de alcohol. Además se encontraron efectos significativos del consumo de alcohol relacionados a la edad, edad de inicio de consumo de alcohol, cantidad de bebidas ingeridas, edad de inicio de consumo de marihuana, y horas transcurridas del consumo de marihuana hasta el accidente $\left(F_{7,116}=15,519, p<0,00\right)$ con una varianza explicada de $40 \%$.

Descriptores: Accidentes; Alcoholismo; Drogas ilícitas; Enfermería de Urgencia.

\footnotetext{
${ }^{1}$ Maestría en Ciencias de Enfermería, Profesora, Facultad de Enfermería, Universidad Autónoma de Nuevo León, México. E-mail: norisnelly@hotmail.com.

${ }^{2}$ Enfermera, Doctor en Enfermería, Profesor Doctor, Escola de Enfermagem de Ribeirão Preto, Universidade de São Paulo, Centro Colaborador de la OMS para el Desarrollo de la Investigación en Enfermería, Brasil. E-mail: macdalri@eerp.usp.br.

${ }^{3}$ Doctor en Filosofía con Espacialidad en Psicología, Profesora de tiempo completo, Facultad de Enfermería, Universidad Autónoma de Nuevo León, México. E-mail: maalonso@fe.uanl.mx.

${ }^{4}$ Doctor en Enfermería con Especialidad en Enfermería Psiquiatría, Profesora de tiempo completo, Facultad de Enfermería, Universidad Autónoma de Nuevo León, México. E-mail: kslg2001@hotmail.com.
}

Correspondencia:

Maria Célia Barcellos Dalrí

Universidade de São Paulo. Escola de Enfermagem de Ribeirão Preto

Av. Bandeirantes, 3900

Bairro Monte Alegre

CEP: 14040-902 Ribeirão Preto, SP, Brasi

E-mail: macdalri@eerp.usp.br 


\title{
Acidentes e lesões por consumo de álcool e drogas em pacientes atendidos em uma sala de urgência
}

Os propósitos deste estudo foram descrever a diferença do consumo de álcool por sexo, escolaridade e ocupação e conhecer o efeito das características sociodemográficas sobre o consumo de álcool, em 120 pacientes. Os resultados mostraram diferenças significativas por sexo $(t=-3.570 ; p<0,01)$ e escolaridade $(t=2,636 ; p<0,01)$ com o consumo de álcool. Ademais, foi encontrado efeito significativo da idade, idade de início de consumo de álcool, quantidade de bebidas ingeridas, idade de início de consumo da maconha e horas decorridas do consumo de maconha ao acidente $\left(F_{7,116}=15.519 ; p<0,00\right)$ sobre o consumo de álcool com variância explicada de $40 \%$.

Descritores: Acidentes; Alcoolismo; Drogas Ilícitas; Enfermagem em Emergência.

\section{Accidents and Injuries Due to Consumption of Alcohol or Drugs in Patients Treated at an Emergency Room}

\begin{abstract}
The aims of the study were to describe the differences in alcohol consumption by sex, education and occupation and to understand the effects of the socio-demographic characteristics on alcohol consumption, in 120 emergency room patients. The results showed significant differences for sex $(t=-3.570, p<.01)$ and education $(t=2.636$, $p<.01$ ) in alcohol consumption. In addition the factors; age, age alcohol consumption started, quantity of alcohol ingested, age marijuana consumption started, and hours lapsed between the consumption of marijuana and the accident $\left(F_{7,116}=15.519, p<.00\right)$, were also significant on the consumption of alcohol with an explained variance of $40 \%$.
\end{abstract}

Descriptors: Accidents; Alcoholism; Street Drugs; Emergency nursing.

\section{Introducción}

En México el consumo de alcohol y drogas ilícitas constituye un preocupante problema de salud pública, lo que se refleja en forma directa e indirecta en las principales causas de mortalidad, como cirrosis hepática, lesiones, accidentes auto infligidos, y accidentes provocados por vehículos motorizados ${ }^{(1-2)}$.

El consumo de alcohol y otras drogas ilícitas ha aumentado progresivamente en los últimos años y se ha relacionando con el ingreso de personas a los centros hospitalarios por hechos vinculados al consumo de estas sustancias, a causa de lesiones intencionales, y a la violencia social y doméstica(3-4).

Diversos estudios han informado que de acuerdo a la droga que se consume es el tipo de lesión que se presenta, además las personas que buscan atención médica en las salas de urgencia por accidentes o lesiones y que han consumido alcohol u alguna otra droga ilícita son menores de 39 años en su mayor proporción, cuentan con una pareja, con un nivel de escolaridad básico o medio básico y trabajan como empleados u obreros $^{(3-7)}$.

Así mismo se señala que la edad de inicio del consumo de alcohol es en promedio a los 17 años, consumen en promedio 10 bebidas alcohólicas y hasta un máximo de 35 a 45 bebidas estándar en un día típico, también se ha documentado que se presenta, en promedio, hasta 8 bebidas estándar el día del accidente, con un máximo de entre 30 y 40 bebidas, por lo que estas personas que presentan accidente o lesión ya poseen problemas en su forma de beber, señalizando un tipo de consumo de alcohol dañino. De acuerdo a los informes se observa que transcurre un promedio de cuatro a seis horas hasta el momento en que sucede el accidente o lesión y la mayor proporción de ellos ya han presentado, en el pasado, por lo menos un accidente ${ }^{(5-7)}$.

Se ha informado que las personas que consumen drogas ilícitas (cocaína, marihuana, inhalables) y han presentado algún accidente o lesión, también presentan 
consumo de alcohol; siendo que el consumo de estas drogas ilícitas se inicio, en promedio, entre las edades de 12 a 17 años ${ }^{(8-9)}$.

En los últimos años, en México, se han realizado estudios acerca del papel del consumo de alcohol en la ocurrencia de accidentes y de violencia; sin embargo existe escasa literatura que aborde la relación del consumo de alcohol y drogas ilícitas con los accidentes y la violencia que se generan en el abordaje del cuidado de la salud en la salas de urgencias. Las salas de urgencias brindan atención permanente a personas que presentan problemas críticos con su salud física y a otras personas que requieren de un tratamiento básico debido a lesiones por accidente y agresiones físicas ${ }^{(10)}$.

Las salas de urgencia son escenarios donde ingresan pacientes con mayor porcentaje de ingesta de alcohol, drogas ilícitas y problemas relacionados al consumo de estas sustancias; esta atención tienen un impacto en la motivación al cambio del consumo de alcohol y drogas ilícitas, por lo que se ha considerado como un lugar ideal para llevar a cabo cualquier tipo de prevención e intervención en las personas alcoholizadas ${ }^{(11)}$.

Asimismo el objetivo de este estudio fue: 1 determinar el tipo de consumo de alcohol y drogas ilícitas y los motivos de ingreso a las salas de urgencias; 2 determinar la cantidad de consumo de alcohol y drogas ilícitas previamente al accidente y lesión; 3- identificar en que etapa de cambio se encuentran los pacientes que sufrieron accidentes y lesiones según el consumo de alcohol y drogas ilícitas; y, 4- describir la diferencia del consumo de alcohol por edad, género, escolaridad y ocupación.

\section{Metódos}

El diseño del estudio fue descriptivo correlacional. El universo estuvo conformado por una población de personas mayores de 18 años que sufrieron accidentes, lesiones y consumieron alcohol o alguna droga ilícita previamente al accidente o lesión y que demandaron atención de salud de un hospital de Seguridad Social del estado de Nuevo León, México.

El muestreo fue por conveniencia; el tamaño de la muestra se obtuvo con un límite de error de estimación de 0,05 , con un nivel de confianza de $95 \%$, para una correlación alternativa de 0,20, para una potencia de $96 \%$. El tamaño de la muestra fue de 120 personas que sufrieron accidentes o lesiones y que fueron admitidas para recibir atención médica en las salas de urgencia.

Se acudió a la sala de urgencia de una institución de Seguridad Social de Nuevo León, México, los fines de semana, se captaron todos los pacientes que ingresaron por haber sufrido un accidente o lesión, que hubiesen consumido alcohol o drogas ilícitas, de ambos géneros, que fuesen mayores de edad, que las lesiones sufridas no requirieran observación en unidad de cuidados intensivos, que no hubiesen provocado la muerte a otros, que se encontrasen clínicamente en condiciones de responder a las preguntas que se les hiciese durante su estancia hospitalaria, que la valoración de la escala de coma de Glasgow, tuviese un puntaje entre 13 y 15 y que aceptasen firmar el consentimiento informado.

Los instrumentos que se utilizaron en la presente investigación fueron:

a) Cuestionario de Factores Sociodemográficos e Historia de Ingreso Hospitalario y Consumo de Alcohol y Drogas (CFSHIHCAD), elaborado por los autores, el cual consta de 21 preguntas divididas en cuatro grupos; en el primer grupo constan la historia de ingreso hospitalario, el motivo de ingreso a la sala de urgencias, el diagnóstico médico, y los ingresos al hospital por causa de accidentes por consumo de alcohol o drogas; el segundo grupo se refiere a factores sociodemográficos como edad, género, estado civil, escolaridad, ocupación, e ingreso económico; en tercer grupo constan la historia de consumo de alcohol, la edad de inicio, la cantidad de copas, el tipo de bebida, el consumo de alcohol antes del accidente o lesión, y la cantidad de bebidas consumidas antes del accidente; el cuarto grupo se refiere a la historia de consumo de drogas ilícitas, a la prevalencia de consumo de drogas ilícitas, al tipo de drogas, a la edad de inicio de consumo, a la cantidad de consumo en un día típico, y a la frecuencia de uso antes del accidente/lesión.

b) La evaluación del cambio Using Alcohol Version of the University Rhode Island Change Assessmente - URICA(12), que consta de 32 ítems tipo escala de Likert que evalúan las actitudes y comportamientos que corresponden a las cuatro etapas de cambio (precontemplación, contemplación, acción y mantenimiento). Este instrumento ha informado una consistencia interna aceptable a través de la prueba de Kudder Richardson.

c) La Prueba de Identificación de Trastornos por Uso de Alcohol de De la Fuente, Kershenobich (OPS/OMS) (AUDIT) (1992)(13). Este cuestionario fue probado en una población Mexicana con una sensibilidad de $80 \%$ y una especificidad de $89 \%$, con un valor promedio predictivo positivo de $60 \%$ y un valor promedio predictivo negativo de $95 \%$. Consta de 10 ítems y examina el uso de alcohol durante los últimos 12 meses y los problemas que 
ocurrieron a partir de la bebida, está constituido por tres dominios, de la pregunta número 1 a la 3 se refieren a la frecuencia y cantidad del consumo de alcohol; se usa para determinar si la persona está bebiendo más del límite sensato y explora el área de lo que podría llamarse como excesivo. Los ítems del 4 al 6, exploran la posibilidad de que exista dependencia del alcohol; y, los ítems del 7 al 10 se refieren a un consumo dañino de alcohol. Si la persona obtiene más de 8 puntos en el AUDIT, se considera que tiene problemas con su forma de beber. El instrumento se ha aplicado en diferentes grupos poblacionales, en trabajadores de la petroquímica del sur de Veracruz y en trabajadores de empresas maquiladoras.

El estudio siguió lo dispuesto en el Reglamento de la Ley General de Salud en Materia de Investigación para la Salud $(1987)^{(14)}$.

\section{Resultados}

De acuerdo a los factores sociodemográficos de la población de estudio, se encontró que un $83,3 \%$ fueron del género masculino, el 48,3\% mencionó ser empleado y el $37,5 \%$ dijo ser obrero; respecto al estado marital el $56,7 \%$ manifestó tener pareja.

En cuanto a la media de edad de los participantes, esta fue de 30,3 años, con una edad mínima de 18 años y una edad máxima de 79 años; respecto al nivel educativo, presentaron una media de 9,5 años de estudio, lo que equivale a un nivel de estudios secundarios, en cuanto al ingreso económico presentaron una media de $\$ 3171,7$ pesos, lo que equivale a cuatro salarios mínimos, con un ingreso mínimo de $\$ 950$ pesos y un ingreso máximo de $\$ 12.500$ pesos, en cuanto a los accidentes previos por consumo de alcohol y drogas, los participantes presentaron en promedio un accidente, con un valor mínimo de uno y un valor máximo de cinco accidentes.

Para verificar el cumplimiento del primer objetivo se utilizaron frecuencias y porcentajes; se observa en la Tabla 1, que el tipo de accidentes que presentaron los participantes que sufrieron accidente y lesiones por consumo de alcohol y drogas tuvo la misma proporción para accidentes automovilísticos y caídas desde su propia altura (34,6\% respectivamente) y riñas $(31,6 \%)$. Los hallazgos muestran que los pacientes que sufrieron accidentes y lesiones consumieron alcohol, mientras que el 14,2\% consumió algún tipo de droga ilícita como marihuana, cocaína e inhalables.

Sobre el tipo de bebida alcohólica que consumieron los participantes antes de presentar el accidente y lesión, la mayor proporción consumió cerveza $(89,2 \%)$, el $1,7 \%$ consumieron brandy y bebidas preparadas, el $3,3 \%$ consumieron tequila, cabe mencionar que el $4,1 \%$ de los participantes bebieron cerveza y tequila. Respecto al tipo de droga que consumieron los pacientes antes del accidente o lesión, en mayor proporción se encuentra la marihuana $(12,5 \%)$, seguida de la cocaína $(3,4 \%)$; además, se observa que el $0,8 \%$ de los pacientes consumió inhalables y que el $2,5 \%$ combinaron el uso de marihuana y cocaína.

De acuerdo al consumo de alcohol el 75\% (IC 95\%, $0,67-0,83$ ) de los participantes que sufrieron accidentes y lesiones presentaron un consumo de alcohol dañino, el $13,3 \%$ (IC 95\%, 0,07-0,19) tuvieron un consumo de alcohol dependiente, mientras que solo el 12\% (IC 95\%, $0,05-0,17)$ presento un consumo sensato de alcohol.

Respecto al tipo de lesión que presentaron los pacientes que sufrieron accidentes y lesiones, se encontró que el $36,7 \%$ presentaron contusiones, seguidos por los politraumatismos $(28,3 \%)$, fracturas (20\%) y en menor proporción fueron las heridas y lesiones (15\%). Así mismo, el 61,6\% de los participantes del estudio, consideran que el evento que presentaron fue un accidente, mientras que el $34,2 \%$ reconocen que sufrieron agresión por otra persona y en menor proporción, el 4,2\% mencionan que el evento que presentaron fue agresión así mismo.

Tabla 1 - Tipo de accidente y lesión que presentaron los participantes por consumo de alcohol y drogas. Nuevo León, 2008

\begin{tabular}{lcc}
\hline \multicolumn{1}{c}{ Variable y Motivo de Ingreso } & f & \% \\
\hline Accidentes automovilísticos & 41 & 34,2 \\
Caídas de su propia altura & 41 & 34,2 \\
Riñas & 38 & 31,6 \\
Tipo de lesión & & \\
$\quad$ Contusiones & 44 & 36,7 \\
Politraumatismo & 34 & 28,3 \\
Heridas y lesiones & 18 & 15,0 \\
Fracturas & 24 & 20,0 \\
\hline
\end{tabular}

Fuente: CFSHIHCAD

$\mathrm{n}=120$

Para verificar el cumplimiento del segundo objetivo, se obtuvieron frecuencias y proporciones; se observó que la media de edad de inicio de consumo de alcohol fue de 17 años de edad ( $D E=2,4)$, con un valor mínimo de inicio de consumo de 10 años y un máximo de edad de 25 años. En promedio consumen 10 bebidas en un día típico, con un valor mínimo de una bebida y un valor 
máximo de 45 bebidas en un día típico. El día del accidente consumieron en promedio 8,9 bebidas alcohólicas, con un valor mínimo de una bebida y un valor máximo de 35 bebidas, cabe señalar que transcurrieron cuatro horas en promedio desde el inicio de consumo al momento del accidente.

La edad de inicio de consumo de marihuana fue a los 16,4 años; en promedio consumen 2,1 cigarros de marihuana en un día típico y habían transcurrido 2,5 horas desde que inició el consumo de la sustancia hasta que sucedió el accidente. Respecto al consumo de cocaína, en promedio lo iniciaron a los 17,7 años de edad; consumen 3,5 inhaladas en un día típico y habían transcurrido entre cuatro y ocho horas antes de que sucediera el accidente. De acuerdo al consumo de inhalables, solo un participante reportó consumirlos antes del accidente, el inicio del consumo sucedió a los 15 años y había transcurrido una hora desde que inició a inhalar hasta el momento del accidente.

En relación al tercer objetivo, se informa que el $51,7 \%$ de los pacientes, que tuvieron accidente por causa del consumo de alcohol, se encuentran en la etapa de contemplación al cambio de consumo y el 34,2\% en la etapa de precontemplación al cambio de consumo. De acuerdo al consumo de drogas la mayoría se encuentran en la etapa de precontemplación y contemplación (52,9\% y $23,5 \%$ ). Los participantes del estudio presentaron la media más alta en la etapa de contemplación $(\bar{\chi}=54,8)$, seguida de la etapa de precontemplación $(\bar{\chi}=47,4)$, de mantenimiento $(\bar{\chi}=45,4)$ y acción $(\bar{\chi}=43,9)$.

Para dar respuesta al cuarto objetivo que señala describir la diferencia del consumo de alcohol por edad, género, y escolaridad, se realizo la prueba estadística inferencial $t$ de Student para diferencia de medias. Se encontró que la variable género $(t=-3,570, p<0,001)$ y escolaridad ( $t=2,636, p<0,001)$ obtuvieron diferencias significativas con el consumo de alcohol. También se observa que las medias de consumo de alcohol entre los grupos de edad y ocupación son diferentes, pero esta diferencia no fue suficiente para ser significativa.
Para observar la asociación del consumo de alcohol con las variables de estudio se realizó el Coeficiente de Correlación de Pearson donde se observó que existe correlación positiva y significativa del consumo de alcohol con la edad $(r=0,19, p<0,05)$, con la cantidad de copas consumidas en un día típico $(r=0,34, p<0,01)$, y con la cantidad de copas ingeridas antes del accidente ( $r=41, p<0,01)$; además se encontró una asociación negativa y significativa del consumo de alcohol con los años de estudio ( $r=-0,21, p<0,05)$, con la edad de inicio de consumo de alcohol $(r=-0,35, p<0,01)$ y con marihuana $(r=-0,23, p<0,01)$, con la cantidad de consumo de marihuana $(r=-0,23, p<0,01)$ y con las horas transcurridas desde el consumo de marihuana hasta que sucedió el accidente $(r=-0,23, p<0,01)$.

Para verificar que si las características sociodemográfica tienen efecto sobre el consumo de alcohol, se utilizó un Modelo de Regresión Lineal Múltiple, se aprecia que el nivel en que las variables edad, edad de inicio de consumo de alcohol, bebidas ingeridas antes del accidente, edad de inicio de consumo de la marihuana y cantidad de uso de la marihuana en un día típico, afectan el consumo de alcohol; el modelo en conjunto fue significativo con una varianza explicada de $40 \%\left(\mathrm{~F}_{7,116}=\right.$ $5,841, p<0,001)$, como se observa en la Tabla 2 y 3.

Tabla 2 - Modelo de Regresión Lineal Múltiple para edad, edad de inicio de consumo de consumo de alcohol, bebidas ingeridas antes del accidente /lesión, edad de inicio de consumo de la marihuana, cuantas veces, en un día típico, usa marihuana conjuntamente con el consumo de alcohol (procedimiento Backward). Nuevo León, 2008

\begin{tabular}{lccccc}
\hline $\begin{array}{l}\text { Fuente de } \\
\text { Variación }\end{array}$ & $\begin{array}{c}\text { Suma de } \\
\text { Cuadrados }\end{array}$ & gl & $\begin{array}{c}\text { Cuadrado } \\
\text { Medio }\end{array}$ & F & Valor de p \\
\hline Regresión & 16054,287 & 5 & 3210,857 & 9,796 & 0,001 \\
Residual & 37364,411 & 114 & 327,758 & & \\
\hline
\end{tabular}

Fuente: CFSHIHCAD, AUDIT

$\mathrm{R}^{2}{ }_{\text {ajustada }}=40 \% \mathrm{n}=120$

Tabla 3 - Contribución de las variables al Modelo de Regresión Lineal Múltiple. Nuevo León, 2008

\begin{tabular}{|c|c|c|c|c|}
\hline \multirow{2}{*}{ Variable } & \multicolumn{2}{|c|}{ Coeficiente no estandarizado } & \multirow{2}{*}{$\mathbf{t}$} & \multirow{2}{*}{ Valor de $p$} \\
\hline & B & Error estándar & & \\
\hline (Constante) & 128,042 & 46,319 & 2,764 & 0,007 \\
\hline Edad & 0,667 & 0,156 & 4,264 & 0,000 \\
\hline Edad de inicio de consumo de alcohol & $-2,880$ & 0,662 & $-4,354$ & 0,000 \\
\hline Cuantas bebidas ingirió antes del accidente & 1,065 & 0,268 & 3,974 & 0,000 \\
\hline Edad de inicio de consumo de la marihuana & $-8,541$ & 3,309 & $-2,581$ & 0,011 \\
\hline En un día típico cuantas veces usa la marihuana & 7,237 & 2,825 & 2,562 & 0,012 \\
\hline
\end{tabular}

Fuente: CFSHIHCAD, AUDIT

$\mathrm{n}=120$ 


\section{Discusión}

El presente estudio reveló que el motivo de ingreso de los pacientes que tuvieron accidentes y lesiones por consumo de alcohol y drogas, que acudieron a salas de urgencias de un hospital de Seguridad Social de Nuevo León México, y se presentaron en mayor proporción, tuvo como causa los accidentes automovilísticos y las caídas de su propia altura (34,2\%) respectivamente; estos datos concuerdan con estudios de investigación ${ }^{(5,15)}$ que informaron resultados similares a los encontrados en este estudio.

Así mismo los tipos de lesiones que presentaron los participantes del estudio fueron en mayor proporción las relacionadas a contusiones, seguidos por politraumatismos, fracturas y en menor proporción fueron las heridas y lesiones; estos datos encontrados son similares a lo informado por otros investigadores ${ }^{(3,5,8,16)}$. Las lesiones ocurren como fenómenos multicausales, que deben ser tratados como un problema de salud pública, no sólo por su influencia en el deterioro de las relaciones sociales y productivas, sino porque producen daños a la salud(16).

En cuanto al tipo de agresión, los participantes reconocen que en mayor proporción el evento presentado, fue producto de un accidente, seguida por agresión por otra persona y en menor proporción por agresión así mismo.

De acuerdo a los resultados encontrados en este estudio las personas que se involucraron con el consumo de sustancias y presentaron accidentes y lesiones son en mayor proporción los participantes del género masculino $(83,3 \%)$, los que mencionan tener pareja, ser empleado y ser menores de 39 años. Otros estudios ${ }^{(3,5)}$ presentan resultados similares a los encontrados, los que muestran que el consumo de alcohol aumenta el riesgo de sufrir un accidente y por consecuencia una lesión; es en este grupo de personas donde predominan los accidentes, donde el accidentado corre el riesgo de sufrir daños que pueden ser irreversibles; además, este grupo de edad es parte de la fuerza productiva del país, esto lleva a reflexionar acerca de las perdidas sociales y económicas que representan los accidentes y lesiones causados por el consumo de alcohol y drogas ilícitas, así es prioritario implementar medidas preventivas dirigidas a este grupo de personas.

Respecto al consumo de drogas lícitas e ilícitas se encontró que todos los pacientes que sufrieron accidentes y lesiones consumieron alcohol, mientras que solo el $14,2 \%$ de ellos consumió algún tipo de droga ilícita, estos datos concuerdan con otros estudios ${ }^{(1,8)}$ que obtuvieron resultados similares. El consumo de alcohol se asocia con el riesgo de sufrir un accidente y con la gravedad de los accidentes sufridos en carretera.

De los que participaron en el estudio, el 14,2\% combinó dos o tres drogas; en mayor proporción se encuentra la marihuana, seguida de la cocaína, en menor proporción los inhalables, además el 2,5\% eran usuarios de marihuana y cocaína; otros autores ${ }^{(1)}$ mencionan a la cocaína con la mayor proporción. Cabe destacar que si bien el alcohol es sumamente peligroso para los conductores, el abuso de las drogas incrementa los efectos en gran medida por la elevada toxicidad. La marihuana altera la noción del tiempo, espacio y atención en los individuos consumidores y la cocaína combinada con el alcohol da como resultado déficit de atención, orientación y de asimetrías sensoriales, observándose deterioros más evidentes en la medida en que aumenta el consumo de esta sustancia, llegando a imposibilitar el adecuado funcionamiento de la persona. Por otro lado respecto al consumo de alcohol el $75 \%$ de los participantes que sufrieron accidentes y lesiones presentaron un consumo de alcohol dañino, seguidos de un consumo de alcohol dependiente y en menor proporción se presentó el consumo sensato de alcohol. Los pacientes que ingresan a las salas de urgencia por presentar accidente o lesión como consecuencia del consumo de alcohol ya presentan problemas con su forma de beber, esto concuerda con resultados de otros investigadores ${ }^{(5-7)}$.

Se encontraron diferencias significativas por género siendo los hombres los que más consumieron y se involucraron en accidentes y lesiones, al contrario de las mujeres; así mismo las personas que cuentan con nivel básico y medio básico de escolaridad consumen más que quienes cuentan con un nivel superior de educación. Datos similares informan diferentes estudios de investigación ${ }^{(3,5,8)}$. Es factible que esto se deba a las normas sociales y culturales de México que permiten que el varón se embriague los fines de semana y es mal visto que una mujer beba en exceso. La mayoría de los participantes que presentaron accidente y lesión se encontraron en la etapa de contemplación al cambio de consumo de alcohol y drogas ilícitas, lo que coincide con otros estudios $^{(6,8)}$ que informaron resultados similares.

El fenómeno del consumo de las drogas y alcohol es un serio problema en el contexto mundial, ya que actualmente los países enfrentan dificultades para el control de producción, comercio y reducción de la demanda de esas sustancias ${ }^{(17)}$. 
Se encontró que los factores edad, escolaridad, edad de inicio del consumo de alcohol y marihuana, $y$ el consumo de alcohol y marihuana antes del accidente mostraron efecto significativo sobre el consumo de alcohol; estos hallazgos son similares a otros estudio(5-7). Es urgente que se establezcan estrategias especificas de intervención para la reducción del consumo de alcohol y drogas en este grupo especifico y que el estudio reveló que, en promedio, habían sufrido un accidente en el pasado y este era su segundo accidente, incrementando con ello el riesgo de consecuencias fatales.

\section{Conclusiones}

Los resultados presentados permiten conocer la información sobre los tipos de consumo de alcohol, drogas ilícitas y motivos de ingreso de pacientes que sufrieron accidentes y lesiones por consumo de alcohol y drogas ilícitas, atendidos en una sala de urgencias.

Cabe destacar que se encontraron diferentes perspectivas de las personas que presentaron accidentes y lesiones, en lo que se refiere a edad, género, escolaridad y ocupación, atendidos en las salas de urgencia, y a pesar de los esfuerzos realizados en el país, los resultados de este estudio muestran que se debe trabajar más sobre la prevención de factores de riesgo y fortalecer los factores protectores de los individuos, de la familia y de las comunidades.

Desde la década anterior del siglo XX hasta este nuevo milenio, existe una preocupación, por parte de las autoridades gubernamentales y las organizaciones nacionales e internacionales de salud, por encontrar estrategias para solucionar este problema. Por esto el abuso de drogas pasa a ser un tema primordial en la agenda nacional e internacional, debido a los daños que causan en los aspectos sociales, económicos y políticos de los países.

Así mismo es importante analizar los cambios que deben realizarse en esta problemática de un modo realista y objetivo, tanto a partir de datos epidemiológicos de investigaciones, como de la experiencia acumulada por las personas envueltas en esta situación, que son las que viven la magnitud y consecuencia del fenómeno causado por las drogas y el alcohol. Por lo tanto, el contexto actual requiere de profesionales especializados, capases de analizar problemas y presentar soluciones y/o estrategias con perspectivas nacionales e internacionales, basadas en datos científicos y también en la unión de esfuerzos con representantes de todos los sectores políticos, de salud y de educación, para promover la participación conjunta con la comunidad a través de programas que tratan los factores de riesgo y aumentan los factores de protección de la sociedad.

La enfermera es el profesional de la salud que puede ocupar un espacio importante en el diseño de políticas públicas en las salas de urgencias. Las habilidades y actividades de la profesión están implícitas en la acción del profesional, como son su capacidad para dirigir y articular formas innovadoras e integradoras para la enseñanza, la investigación y promoción de la salud en el contexto de los accidentes relacionados con el alcohol y las drogas, lo que establece que las enfermeras, en el escenario de la sala de urgencias, tengan una práctica sustentada en el conocimiento técnico y científico y actitudes flexibles y sensibles en el universo de su trabajo.

\section{Agradecimientos}

Agradecemos a la Comisión Interamericana para el Control del Abuso de Drogas/CICAD de la Secretaria de Seguridad Multidimensional/SSM de la Organización de los Estados Americanos/OEA, la Secretaria Nacional de Políticas sobre Drogas/SENAD do Gabinete de Seguridad Institucional/Brasil, la Escuela de Enfermería de Ribeirao Preto de la Universidad de Sao Paulo y Centro Colaborador de la Organización Mundial de la Salud para el Desarrollo de la Investigación en Enfermería, la población representada en los estudios de investigación, bien como a las autoridades de las universidades representadas por los participantes del Programa En-Line de Especialización en Investigación sobre el Fenómeno de las Drogas - PREINVEST, periodos 2005, 2006, 2007 y 2008.

\section{Referencias}

1. Pastor R, Liopis JJ, Baquero A. Adiciones. 2003; 15(2):159-64. 2. Estadística de Mortalidad en México: Muertes registradas en el año 2003. Salud Pública México. 2003; 47(2):171-87.

3. Casanova L, Borges G, Mondragón L, Mediana M, Cherpertiel Ch. El Alcohol comoFactor de Riesgo en Accidentes Vehiculares y Peatonales. Salud Mental. 2001; 24(5):3-11.
4. Rosovsky H. Accidentes y Conducta Violenta Asociados a la Ingesta de Alcohol en la Ciudad de México:hacia una Estrategia Preventiva. Ponencia Presentada en la Tercera Reunión Anual del Programa Universitario de Medio Ambiente-UNAM. México (DF): Academia Nacional de Medicina; 1994. p. 19-21. 
5. Flores F, Rodríguez L, Guzmán F. Consumo de Alcohol y Características Demográficas de Usuarios de Salas de Urgencias de Tamaulipas, México. SMAD, Revista Electrónica Salud Mental Alcohol Drog 2006; 2(1):1-14.

6. Alonso CM, Esparza AS. Intervención Breve: Una Estrategia para Disminución del Consumo de Alcohol en Personas que Sufrieron un Accidente Automovilístico. Desarroll Científico Enferm 2003; 11(1):6-11.

7. Oliva RN. Accidentes Automovilísticos y el Consumo de Alcohol. [Tesis de Maestría] Monterrey (NL), Universidad Autónoma de Nuevo León; 2001.

8. Gonzalez JA. Etapas de Cambio en Personas que Sufrieron Accidentes y Lesiones por Consumo de Alcohol y Drogas. [Tesis de Maestría] Monterrey (NL), Universidad Autónoma de Nuevo León; 2002.

9. Piñeiro S, Medina E, Rojas M. Prevalencia del Consumo de Drogas en Pacientes Atendidos en Urgencias de Adultos. Salud Pública México. 1998; 40(3):1-12.

10. Gómez J. Urgencias, gravedad y complejidad: un constructo teórico de la urgencia basada en el triaje estructurado. Emergencias. 2006; (18):156-64.

11. Rumpf $\mathrm{HJ}$, Hapke $U$, Jhon $U$. Previus help seeking and motivation to Change drinking behaviour in alcohol-dependent general hospital patients. General Hospital Psychiatry. 1998; (20):115-9.
12. Diclement $\mathrm{C}$, Hungler $\mathrm{S}$. Stages of Change profiles in alcoholismo treatment. Sustance Abuse. 1990; (2):217-35.

13. De La Fuente J, Kershenobich D. El Alcoholismo como problema Médico. Facultad de Medicina UNAM. 1992; 35(2):47-51.

14. Secretaria de Salud. Reglamento de la ley general de salud en materia de investigación para la salud. México: Editorial Porrua; 1987.

15. Borges G, Mediana MM, Cherpitel Ch, Casanova L, Mondragón L, Romero M. Consumo de Bebidas Alcohólicas en Pacientes de los Servicios de Urgencias de la Ciudad de Pachuca, Hidalgo. Salud Publica México. 1999; 41(1):3-11.

16. Hidalgo SE, Híjar M, Blanco MJ, Kageyama EM. Factores asociados con la gravedad de lesiones ocurridas en la vía pública en Cuernavaca, Morelos, México. Salud Pública México. 2005; 47 (1):30-8.

17. Wright MGM, Gliksman L, Khenti A, Furegato ARF. A pesquisa sobre o fenômeno das drogas na perspectiva dos estudos multicêntricos na América Latina e Caribe. Rev. Latino-Am. Enfermagem. [Internet]. 2009 [acesso em: 11 mar 2010] ; 17(spe):759-61. Disponível em: http:// www.scielo.br/scielo.php?script=sci_arttext\&pid =S0104$11692009000700001 \&$ Ing $=$ pt. doi: 10.1590/S010411692009000700001. 